\title{
Thermodynamic Simulation Modeling Analysis and Experimental Research of Vertical Ultrasonic Vibration Assisted EDM
}

\section{Yinghuai Dong}

Tianjin University of Science and Technology

Jiajun Liu

Tianjin University of Science and Technology

Guangyan Li

Tianjin Institute of Aerospace Mechanical and Electrical Equipment, Tianjin, China

Yan Wang ( $\nabla$ satansdestiny@163.com )

Tianjin University of Science and Technology

\section{Research Article}

Keywords: UEDM, Temperature field model, Material removal rate (MRR), surface topography

Posted Date: June 25th, 2021

DOI: https://doi.org/10.21203/rs.3.rs-634020/v1

License: (9) This work is licensed under a Creative Commons Attribution 4.0 International License. Read Full License

Version of Record: A version of this preprint was published at The International Journal of Advanced Manufacturing Technology on January 15th, 2022. See the published version at https://doi.org/10.1007/s00170-021-08604-z. 


\section{Thermodynamic Simulation Modeling Analysis and Experimental Research of}

Vertical Ultrasonic Vibration Assisted EDM

Yinghuai Dong ${ }^{\text {a.b }}$, Jiajun Liu ${ }^{\mathrm{a}}$, Guangyan Lic, Yan Wang ${ }^{*}$.a.b

${ }^{a}$ Tianjin University of Science and Technology, Tianjin, China

${ }^{b}$ Tianjin Key Laboratory of Integrated Design and On-line Monitoring for Light Industry \& Food Machinery and Equipment, Tianjin, China

${ }^{c}$ Tianjin Institute of Aerospace Mechanical and Electrical Equipment,

Tianjin, China

First author (email: dongyh@tust.edu.cn; Tel: 086-22-60600738)

*Corresponding author (email: satansdestiny@ 163.com; Tel: 086-22-60600738)

Abstract: Compared with traditional EDM, ultrasonic vibration assisted EDM ((UEDM)) shows better performance in machining efficiency and surface quality. But the material removal process of UEDM is complex, and there are many influencing factors, so it is difficult to describe the material removal process accurately. In this study, based on the voltage variation during UEDM processing and combined with the heat transfer theory, the material removal model of TC4 titanium alloy under the condition of single pulse vertical ultrasonic vibration UEDM was established, and the material removal process of UEDM under different amplitudes was analyzed. The machining efficiency and surface quality of UEDM with different ultrasonic energy under the condition of vertical ultrasonic vibration are obtained verified by UEDM experiments. The best ultrasonic energy under different current can be obtained by adjusting the current and ultrasonic vibration energy, which can improve the efficiency of UEDM.

Key words: UEDM, Temperature field model, Material removal rate (MRR), surface topography

\section{Introduction}

EDM is in a certain discharge medium, through the instantaneous pulse discharge at both ends of the electrode, the instantaneous local high temperature is produced in the ion channel, which makes the surface of the workpiece melt or even vaporize, so as to achieve the purpose of material removal on the workpiece surface [1]. Because of the high energy density of discharge, EDM plays a very important role in machining metal materials with high hardness, high melting point, high toughness and brittleness, especially in aerospace, medical devices and other industries. However, in the EDM process, the processing debris gathered in the machining gap, it is easy to produce some bad phenomena, such as secondary discharge, arc discharge and short circuit, etc. [2]. With the increase of machining depth, this phenomenon is more obvious, resulting in the gradual decrease of EDM efficiency with the machining depth. Ultrasonic cavitation can impact and crush debris. Accelerate the circulation of working fluid and improve the stability of discharge. Therefore, researchers are committed to ultrasonic vibration-assisted EDM (UEDM).

UEDM is to apply high-frequency ultrasonic vibration on the electrode or workpiece, using the cavitation and pumping effect of ultrasonic vibration to accelerate the flow of the working liquid in the discharge gap and discharge residue, so as to improve the material removal rate of EDM. Kremer et al. (1989) reviewed the effects of ultrasonic 
vibration on the processing and surface modification of EDM. The results demonstrate that after applying ultrasonic vibration, the MRR increases significantly and the machining process is more stable. Subsequently, it is found that the surface heat-affected layer, thermal residual stress and micro-cracks after ultrasonic vibration-assisted EDM are reduced [3-4]. Lin et al. (2000) adopted the combined process of ultrasonic machining (USM) and electrical discharge machining (EDM) to study the machining characteristics of Ti-6Al-4V. The results show that this combination process can increase the material removal rate and reduce the thickness of the recast layer. In addition, from the voltage waveform analysis, this combination can reduce abnormal discharge and improve discharge efficiency [5]. Abdullah et al. (2009) studied the effect of tool electrode ultrasonic vibration on the surface integrity of tungsten carbide (WC-CO) machined by EDM. The SEM micro-hardness test and morphology analysis show that ultrasonic-assisted EDM can improve the surface integrity [6-7]. Shabgard et al. (2015) machined titanium alloy by UEDM and studied the effects of electrode vibration, current and pulse width on MRR, electrode loss, stability of the machining process and machined surface quality. the results show that high frequency vibration of the electrode reduces micro-crack density and electrode loss in finishing, but increases recast layer thickness, micro-crack density and electrode loss in rough machining [8]. Liu et al. (2018) based on dynamic simulation. A model for three-dimensional (3D) gap flow field is established, and the movement laws of debris with different amplitudes, frequencies and time gaps with different aspect ratios are quantitatively analyzed. The results show that periodic ultrasonic vibration is useful to the removal of debris in the machining gap. Ensure the stability of the machining process and improve the machining efficiency [9]. Wang et al. (2020) studied the material removal mechanism of horizontal UEDM according to the heat transfer theory. By establishing the UEDM single pulse removal volume model, the surface formation process and the influence law of ultrasonic vibration were analyzed, and the correctness of the theoretical derivation was verified by simulation and experiments [1]. Choubey et al. (2019) used two-dimensional axisymmetric FEM to predict the crater shape with and without vibration Micro-EDM, and obtained the surface temperature distribution and material removal rate of the workpiece [10].

Researchers have conducted a more in-depth experimental study on UEDM, but there are relatively few studies on the material removal process and surface formation process of UEDM. In this study, based on the voltage change during UEDM, the mathematical model of UEDM material removal process is established by using EDM heat transfer theory, and the single-pulse UEDM removal volume model is analyzed by ANSYS thermodynamic simulation. The correctness of the theoretical model is verified by experiments, and the best matching ratio between ultrasonic amplitude and EDM process is revealed, which provides a reference for the setting of UEDM parameters.

\section{UEDM mathematical model}

In EDM, the machine tool servo system controls the tool electrode to approach the workpiece electrode. When the gap between the electrodes is certain distance, the charged particles break down the discharge gap and form a discharge channel in the 
medium. Due to the high-speed collision of charged particles in the discharge channel, the energy density of the discharge channel is very high. In addition, the medium has a compression effect on the discharge channel, resulting in a sharp rise in temperature, and the high temperature makes the electrode surface material melt or even vaporize. Part of the metal on the surface of the tool and workpiece is removed, and pits are formed on the surface of the workpiece [2]. Fig. 1 is a schematic diagram of EDM pits.

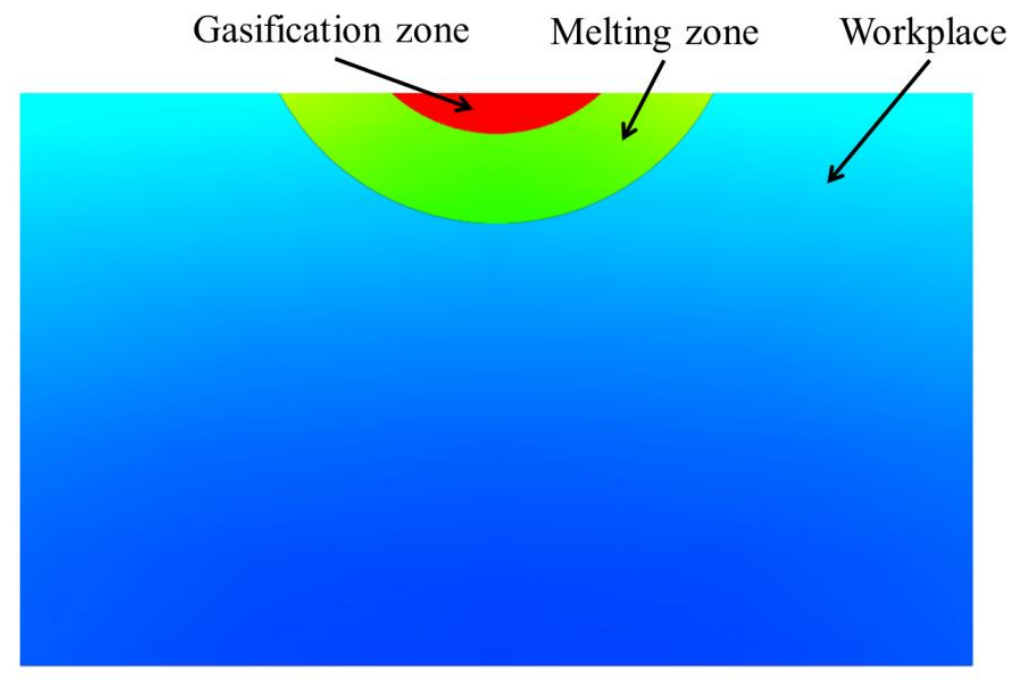

Fig.1 Schematic diagram of pits in EDM

The thermal physical process of ultrasonic assisted pulse EDM is comparable to that of EDM, which is very short and complex. However, different from EDM, the high-frequency vibration of ultrasound will constantly change the discharge gap of EDM, which can reduce short circuit and arc discharge. Ultrasonic cavitation, pumping and eddy current can obviously improve the pulse discharge state. When the binding force between molten metal and liquid molecules is not enough to withstand the interaction pressure caused by ultrasonic high frequency vibration, the molten metal material will be discharged from the discharge gap, which improves the removal rate of processing materials.

\subsection{Mathematical model of UEDM discharge energy}

In the process of EDM, when the inter-electrode medium is broken down, the power supply rapidly releases energy in the discharge channel and converts the electric energy into thermal energy, magnetic energy, light energy, sound energ $\mathrm{y}$ and electromagnetic wave radiation, etc. the total energy generated by the pu lse power supply in the discharge gap is:

$$
W=\int_{0}^{t_{k}} U(t) I(t) d t
$$

The energy $W$ released in the discharge gap includes three parts: the energy $W_{\tau}$ jar released by the discharge passage plasma. The energy $W_{a}$ released by the anode surface, and the energy $\mathrm{W}_{\mathrm{k}}$ of the cathode surface released.

$$
W=W_{\tau}+W_{a}+W_{k}
$$

The energy released by the plasma in the discharge channel can be expressed as 
follows:

$$
W_{\tau}=\int_{0}^{t_{k}} U_{\tau}(t) I(t) d t
$$

Where $U_{\tau}(t)$-Pressure drop $(\mathrm{V})$;

$I(t)$ - discharge current (A);

$t_{k}$ - discharge duration in the discharge channel plasma(s).

When the dielectric property between the electrodes is not destroyed, it is considered that the applied voltage $U$ is uniformly distributed in the discharge medium, and when the distance between the electrodes is $\mathrm{H}_{0}$, the electric field strength in the discharge medium is Elastic $\mathrm{U} / \mathrm{H}_{0}$. When the inter electrode dielectric is broken down and discharged, the intrapolar potential distribution and point field intensity change abruptly [2].

$$
W_{\tau}=\int_{0}^{t_{k}} E_{\tau}(t) H_{0} i(t) d t
$$

where $E$-Electric field strength;

$H_{0}$ - Polar distance.

The discharge gap during the machining process changes with the amplitude of the ultrasonic vibration when the ultrasonic vibration acts on the electrode or the workpiece. In the study of Bai et al, it indicates that the EDM increases with the increase in the gap width, and the diameter and depth of the generated slit will decrease as the gap width increase [11]. In the UEDM processing, due to the high frequency vibration of the ultrasound, the actual discharge clearance varies with the amplitude and frequency of the ultrasound, and therefore, the actual machining gap of the ultrasonic vibration assistant electric spark is:

$$
H=H_{0}+A \sin (2 \pi f t+\varphi)
$$

where $A$-Ultrasonic amplitude;

$F$-Ultrasonic vibration frequency;

$\varphi$-Initial phase.

During the UEDM process, the ultrasound high frequency vibration constantly changes the discharge distance in the electric spark processing, causing the continuous change of the inter-discharge gap results in the variation in the plasma channel (there is research in the following text). The formula of ultrasonic vibration-assisted plasma voltage is as follows:

$$
U_{t}=E_{t}\left(H_{0}+A \sin (2 p f t+\varphi)\right)
$$

Therefore, the energy of the ultrasonic vibration auxiliary electric spark plasma channel discharge is:

$$
W_{t}=\int_{0}^{t_{k}} E_{t}(t)\left(H_{0}+A \sin (2 p w t+\varphi)\right) i(t) d t
$$

\subsection{UEDM heat source model}

Most of the energy released by EDM discharge is in the form of thermal energy, which is finally distributed on the positive and negative electrodes to form an instantaneous high temperature heat source. The heat source on the electrode is 
normally divided into a body heat source and a surface heat source, while the body heat source accounts for only $1 \%$ or $2 \%$ of the surface heat source. Therefore, the heat source generated by the electric spark discharge will be dominated by the surface heat source [2]. The diameter of the heat source area is usually related to the pulse width, open-circuit voltage and peak current. The heat flux density of single pulse spark discharge is Gaussian distribution, and the heat flux equation of a single spark is like equation [12]:

$$
q_{(r)}=\frac{4.57}{\pi R(t)^{2}} \beta \times \mathrm{U}_{\tau}(t) \times I(t) \exp \left(-4.5 \frac{r^{2}}{R(t)^{2}}\right)
$$

Where $\beta$-The energy distribution coefficient;

$U$-The voltage in discharge Channel(V);

$I$-The current in discharge Channel(A);

$R(t)$ - The radius of $t$ discharge channel at time $(\mu \mathrm{m})$.

\subsection{Energy distribution}

In the study of the energy distribution coefficient of bipolar discharge, Dibitoto et al reported that the heat absorption on the cathode and anode is $18.3 \%$ and $8 \%$ respectively, and the rest of the heat is lost to the interstitial fluid [13]. However, in the work of Shabgard et al has been proved that the proportion of heat flux entering the electrode is not constant, but a function related to the input parameters of the process, and the correlation between the pulse current and the pulse conduction time obtained by power regression modeling, such as the formula [14]:

$$
\begin{gathered}
f_{c}=5.5998 \times I^{-0.3401} \times T_{i}^{0.2989} \\
f_{a}=-19.2521+38.8627 \times I^{-0.2008} \times T_{i}^{0.1889}
\end{gathered}
$$

where $f_{\mathrm{c}}$-Cathode energy coefficient;

$f_{\mathrm{a}}$-Anode energy coefficient.

\subsection{Spark discharge radius}

The discharge radius of the spark is also an important factor in the process of simulation modeling. In this study, the radius of the discharge channel (spark) is the spark radius obtained by the work of Ikai and Hashiguchi, which is called the equivalent heat input radius, which depends on the function of pulse current and pulse conduction time [15], as shown in the following equation:

$$
R(t)=2.04 \times I^{0.43} \times T_{i}^{0.44}
$$

where $I$ - the pulse current (A);

$T_{i}$ - The pulse turn-on time $(\mu \mathrm{s})$.

\subsection{Heat transfer model}

Heat transfer in the EDM process is a nonlinear transient heat conduction problem. The Gaussian heat source distribution is applied to the electrode surface heat source for material removal. In this paper, it is assumed that the volume heat source $\mathrm{q}_{\mathrm{v}}=0$, of the workpiece satisfies the Fourier conduction theory, and the differential equation of heat conduction in the cylindrical coordinate system is obtained [16]: 


$$
\frac{1}{r} \frac{\partial}{\partial r}\left(K_{r} \frac{\partial t}{\partial r}\right)+\frac{\partial}{\partial z}\left(K_{r} \frac{\partial t}{\partial z}\right)=\rho C_{p} \frac{\partial T}{\partial t}
$$

where $T$-Temperature $(\mathrm{K})$;

$t$-Time(s);

$\rho$-Density $\left(\mathrm{kg} / \mathrm{m}^{3}\right)$;

$K_{r}$ - Thermal conductivity $\left(\mathrm{W} \cdot \mathrm{m}^{-1} \mathrm{~K}^{-1}\right)$;

$C_{p}$ - Material heat capacity $\left(\mathrm{J} \cdot \mathrm{kg}^{-1} \mathrm{~K}^{-1}\right)$;

$r, z$-The position of the point in the cylindrical coordinate system.

\subsection{Setting of boundary conditions}

The boundary condition is set as shown in the Fig.2. During the electric spark discharge, the heat flux on the top surface of the workpiece in the spark radius "R" melts and even vaporizes the workpiece surface, and contacts with the discharge medium outside the radius $\mathrm{R}$. this paper intends to simulate the heat loss by thermal convection. Boundaries $\mathrm{B}_{2}, \mathrm{~B}_{3}$ and $\mathrm{B}_{4}$ consider that there is no heat transfer between the spark discharge area and infinitely far away from the spark discharge area. Therefore, the loss heat flux is assumed to be zero. When the discharge time $t>0$, the boundary condition is set as follows [14]:

$$
K(\partial T / \partial z)= \begin{cases}h_{c}\left(T-T_{0}\right) & r>R \\ q(r) & r \leq R \\ 0 & \end{cases}
$$

On boundaries $\mathrm{B}_{2}, \mathrm{~B}_{3}$ and $\mathrm{B}_{4}$,

$$
(\partial \mathrm{T} / \partial \mathrm{n})=0
$$

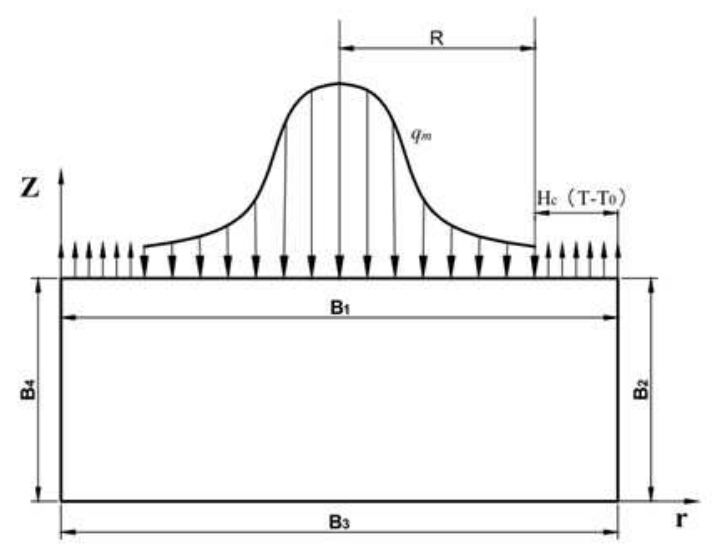

Fig. 2 Boundary conditions of Gaussian heat source distribution

As shown in Fig.2, $\mathrm{q}_{\mathrm{m}}$ is the heat flux entering the workpiece, and $\mathrm{H}_{\mathrm{c}}$ is the convective heat transfer coefficient. In this paper, the discharge medium is kerosene, and the convective heat transfer coefficient is $500 \mathrm{~W} / \mathrm{m}^{2} \cdot \mathrm{k} \sim 1000 \mathrm{~W} / \mathrm{m}^{2} \cdot \mathrm{k} ; \mathrm{T}_{0}$ is room temperature, and the direction $\mathrm{n}$ is perpendicular to the boundary. The initial temperature $\mathrm{T}_{0}$ is taken as the ambient temperature of the dielectrics immersed in it (the initial temperature in this paper is $25^{\circ} \mathrm{C}$ ).

\section{Simulation analysis of single-pulse UEDM}

According to the theoretical derivation of UEDM mentioned above, the transient thermodynamic finite element analysis of single-pulse discharge is carried out, and 
the numerical simulation of ANSYS Workbench and ANSYS parameter design language (APDL) are carried out in this paper.

In this paper, the machined material is TC4 titanium alloy, and its physical properties and related parameters are shown in Table 1. It is known that in the actual EDM process, the maintenance voltage is $25 \mathrm{~V}$, the frequency of the ultrasonic vibration device is $20 \mathrm{kHz}$, and the amplitude range is $0-20 \mu \mathrm{m}$. Therefore, the machining parameters are set as shown in Table 2.

Table 1 Physical property parameters of TC4 Titanium Alloy

\begin{tabular}{ccc}
\hline $\begin{array}{c}\text { Temperature } \\
\mathrm{T} /{ }^{\circ} \mathrm{C}\end{array}$ & $\begin{array}{c}\text { Thermal conductivity } \\
\lambda /\left(\mathrm{W} \cdot \mathrm{m}^{-1} \cdot{ }^{\circ} \mathrm{C}^{-1}\right)\end{array}$ & $\begin{array}{c}\text { Specific heat capacity } \\
\mathrm{c} /\left(\mathrm{J} \cdot \mathrm{kg}^{-1} \cdot{ }^{\circ} \mathrm{C}^{-1}\right)\end{array}$ \\
\hline 25 & 5.42 & 678.13 \\
100 & 6.68 & 690.69 \\
200 & 8.76 & 703.25 \\
300 & 10.43 & 740.92 \\
400 & 12.52 & 753.48 \\
500 & 14.18 & 879.06 \\
600 & 15.86 & 920.92 \\
700 & 15.86 & 926.78 \\
800 & 15.86 & 1000.64 \\
1000 & 15.86 & 1046.50 \\
1660 & 15.86 & 1088.36 \\
\hline
\end{tabular}

Table 2 Simulation parameter setting

\begin{tabular}{|c|c|c|c|c|}
\hline \multicolumn{3}{|c|}{ EDM parameter setting } & \multicolumn{2}{c|}{ Ultrasonic parameter setting } \\
\hline $\begin{array}{c}\text { Current } \\
(\mathrm{A})\end{array}$ & $\begin{array}{c}\text { Voltage } \\
(\mathrm{V})\end{array}$ & $\begin{array}{c}\text { Discharge time } \\
(\mu \mathrm{s})\end{array}$ & $\begin{array}{c}\text { Frequency } \\
(\mathrm{Hz})\end{array}$ & $\begin{array}{c}\text { Amplitude } \\
(\mu \mathrm{m})\end{array}$ \\
\hline 2 & 25 & 50 & 20000 & $0,6,12$ \\
\hline
\end{tabular}

In addition, there are many factors affecting UEDM processing, the model needs to be properly simplified, so the following assumptions are put forward for the model [14]:

1. The model is only for a single pulse UEDM, and the model is central axisymmetric.

2. The electrode in single-pulse EDM is regarded as a semi-infinite body because the volume of the removed material is much smaller than that of the electrode.

3. Ignore the effect of material phase change on the material removal rate in the process of analysis.

4. Neglect the effect of thermal radiation and heat conduction on temperature, because thermal radiation and heat conduction are relatively small relative to heat flux.

5. The heat flux incident on the electrode has nothing to do with the affected surface profile.

\subsection{Simulation results of single-pulse EDM}

In this paper, on the basis of single-pulse EDM temperature field simulation, ultrasonic vibration parameters are added, and the comparison of EDM and UEDM temperature field simulation results is shown in Fig.3. 


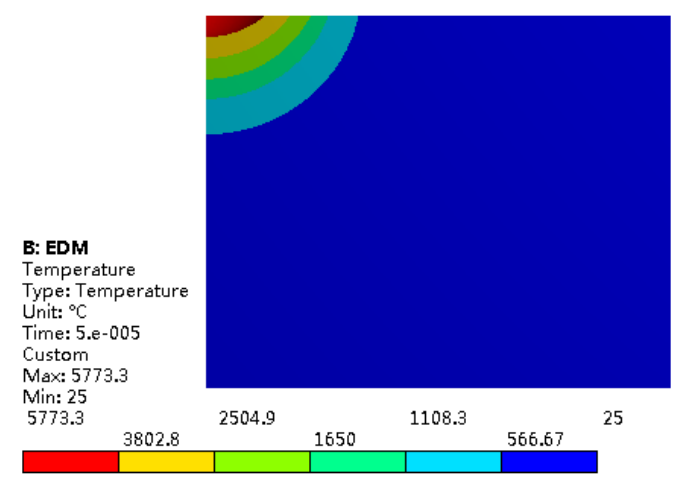

(a) Temperature field

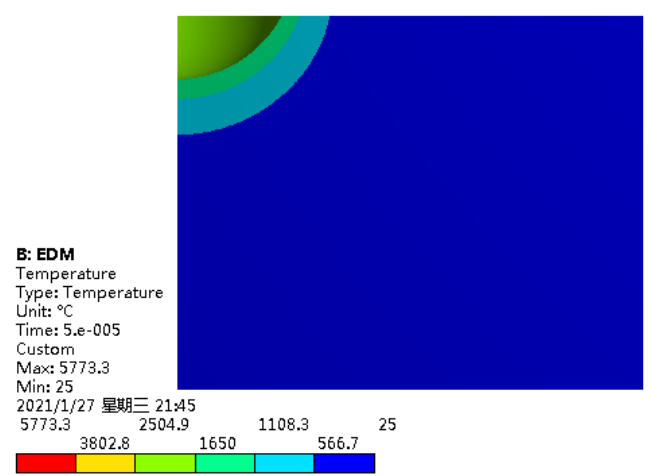

(b) Remove material section view

Fig.3 EDM temperature field distribution cloud diagram and schematic diagram of removing

$$
\text { material }
$$

Through transient thermodynamic analysis of the EDM processing temperature field, it is assumed that all the melting materials are removed, and the simulation results are obtained as shown in Fig. $3 \mathrm{~b}$. The central temperature is $5773.3^{\circ} \mathrm{C}$ and decreases gradually with the increase of radius and depth. The part that reaches the melting temperature will be removed from the discharge gap in the form of debris, and the removal depth of the material is $13.8 \mu \mathrm{m}$.

\subsection{Simulation results of single-pulse UEDM}

UEDM simulation is based on EDM simulation to increase ultrasonic vibration parameters, by changing the discharge gap of EDM to affect the heat flux of discharge gap, and then affect the material removal rate of the workpiece surface. UEDM simulation results are shown in Fig.4.

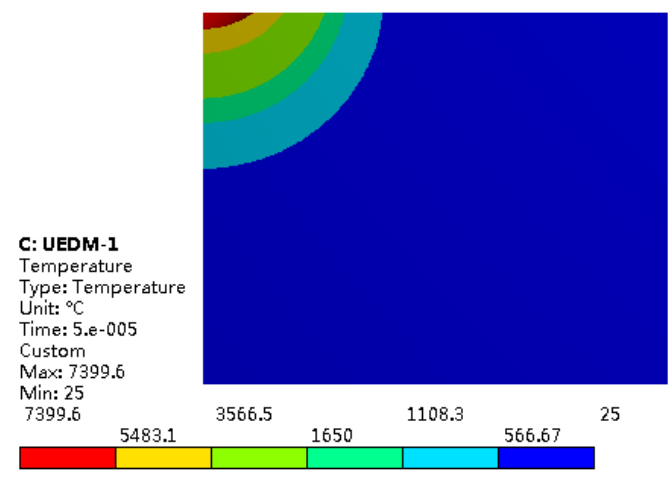

(a) Temperature field

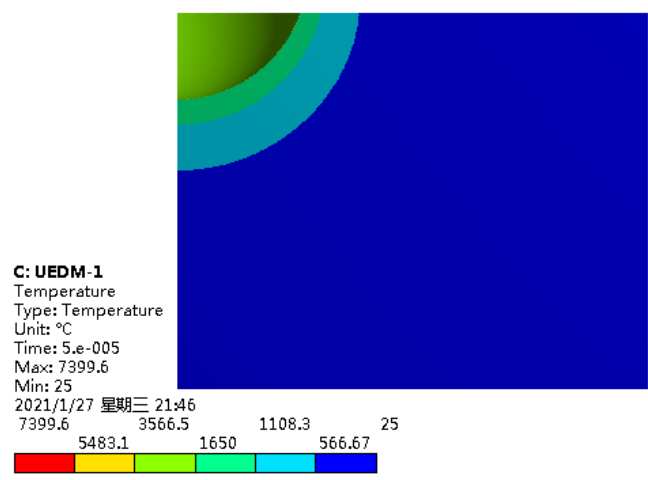

(b)Remove material section view

Fig. $46 \mu \mathrm{m}$ amplitude UEDM temperature field distribution cloud map and schematic diagram of removing material 


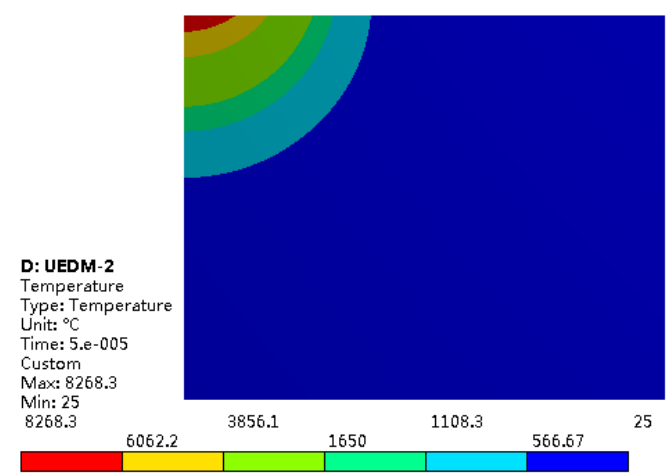

(a) Temperature field

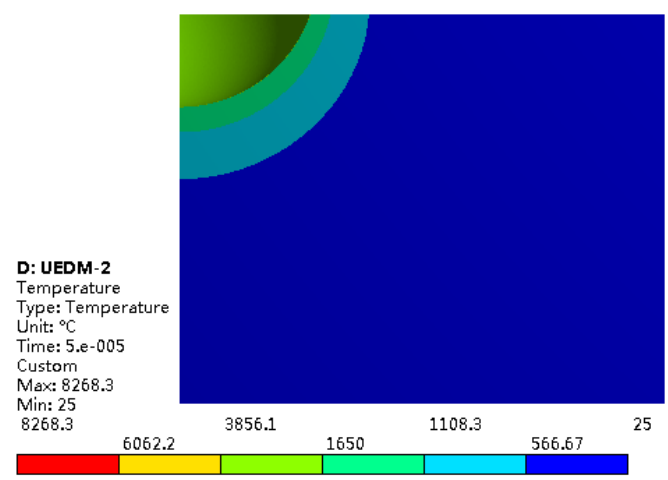

(b)Remove material section view

Fig. $512 \mu \mathrm{m}$ amplitude UEDM temperature field distribution cloud diagram and removal material schematic diagram

By comparing the temperature field distribution cloud images of EDM and UEDM, it is observed that after applying ultrasonic vibration, the surface temperature of the workpiece increases greatly, and the temperature increases gradually with the increase of ultrasonic amplitude, and the material removal volume increases accordingly. When $6 \mu \mathrm{m}$ ultrasonic vibration is applied, the highest temperature is $7399.6^{\circ} \mathrm{C}$ and the material removal depth is $18.8 \mu \mathrm{m}$, as shown in Fig.4. When the ultrasonic amplitude is $12 \mu \mathrm{m}$, the highest temperature is $8268.3^{\circ} \mathrm{C}$ and the material removal depth is $19.4 \mu \mathrm{m}$, as shown in Fig.5.

The reason for the above results is that the high frequency vibration of ultrasound constantly changes the discharge gap of UEDM, so that the gap voltage increases at first and then decreases with the amplitude. The instantaneous heat flux of UEDM increases with the instantaneous increase of voltage, thus the center temperature increases.

\section{Experimental Verification}

\subsection{Test Equipment and Parameter Setting}

In this experiment, UEDM is realized by CHMER-EDM machine tool and self-made ultrasonic shaking table. The oscilloscope (Siglent SDS 1072CFL) is used to detect and collect the voltage waveform between the electrodes, and the microstructure is observed by scanning electron microscope (SEM). The UEDM experimental device is shown in Fig.6.

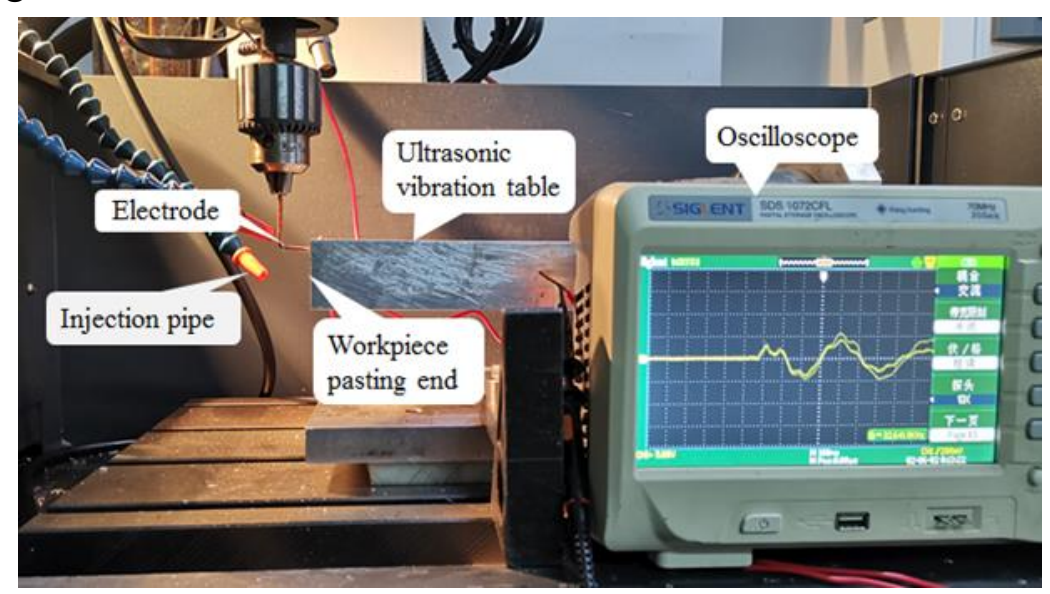


Fig.6 UEDM setting

In order to prove the voltage change of EDM assisted by different ultrasonic amplitudes, experiments are designed to verify the variation characteristics of EDM efficiency with ultrasonic amplitudes under different current and pulse width conditions. Because ultrasonic amplitudes are difficult to be accurately measured, ultrasonic amplitudes are changed by controlling the power of ultrasonic shaking table; UEDM parameters are set as shown in Table 3.

Table 3 Processing parameters

\begin{tabular}{cc}
\hline Processing parameters & Parameter value \\
\hline Ultrasonic vibration frequency $(\mathrm{kHz})$ & 20 \\
Ultrasonic power ratio & $0 \%, 30 \%, 60 \%, 90 \%$ \\
Open circuit voltage ( V) & $240 \mathrm{~V}$ \\
Current (A) & $2 、 4 、 6$ \\
Pulse width (us) & 150 \\
Pulse interval (us) & 50 \\
Discharge gap (um) & 12 \\
\hline
\end{tabular}

In this study, the copper electrode of $\varphi 3 \mathrm{~mm}$ is used, and the workpiece is made of $2 \mathrm{~mm} \times 20 \mathrm{~mm} \times 30 \mathrm{~mm}$ TC4 titanium alloy with a processing depth of $0.2 \mathrm{~mm}$. In UEDM, carbon deposition occurs on the end face of the electrode after each machining, which affects the next processing. So it is necessary to flatten the end face of the motor. Therefore, the electrode surface is finely machined with a micro electric grinding hammer to ensure that the end face of the electrode is smooth and uniform before each machining.

\subsection{Discharge Voltage Waveform Analysis}

In the discharge process, the Discharge voltage waveform during EDM and UEDM are collected, respectively, as shown in Fig.7.

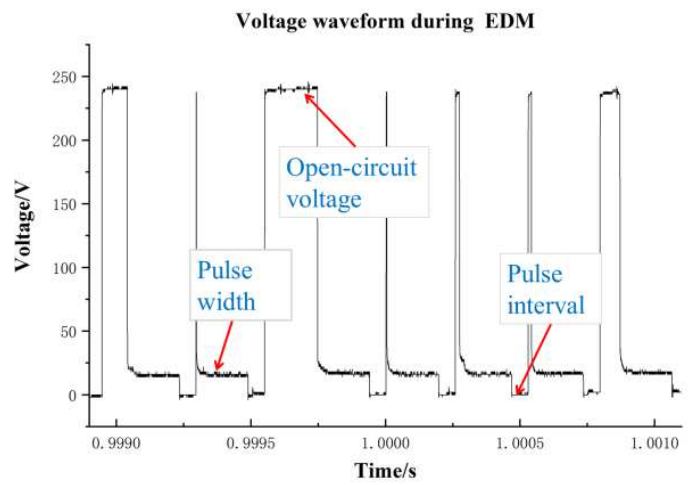

a)EDM Voltage waveform

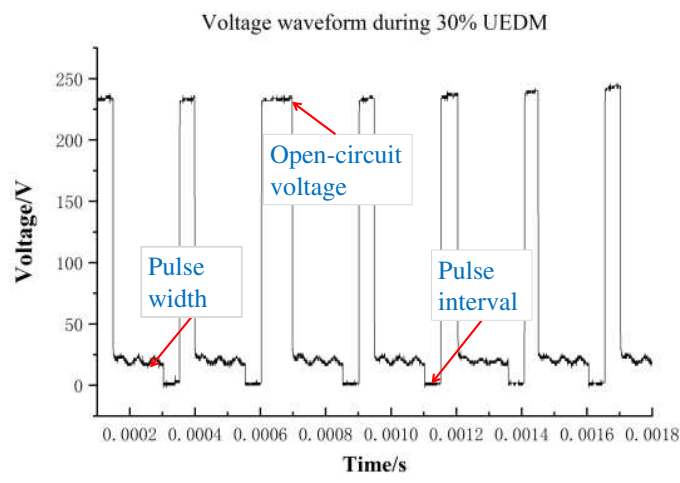

b) UEDM of $30 \%$ ultrasonic power specific voltage waveform 


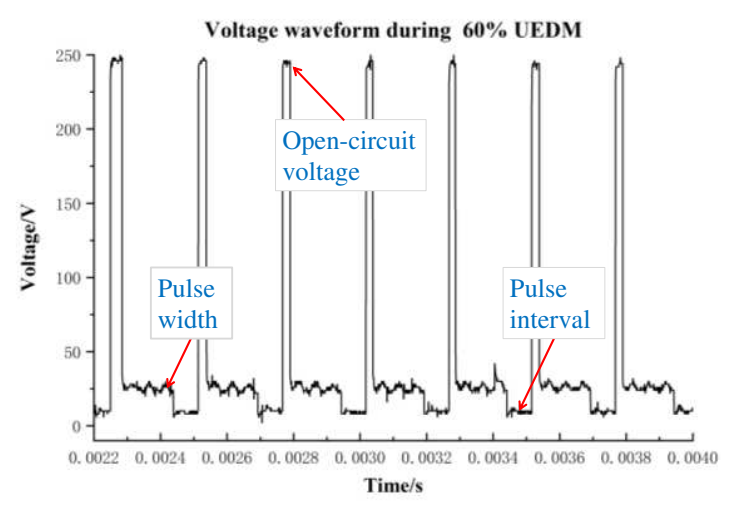

c) UEDM of $60 \%$ ultrasonic power specific voltage waveform

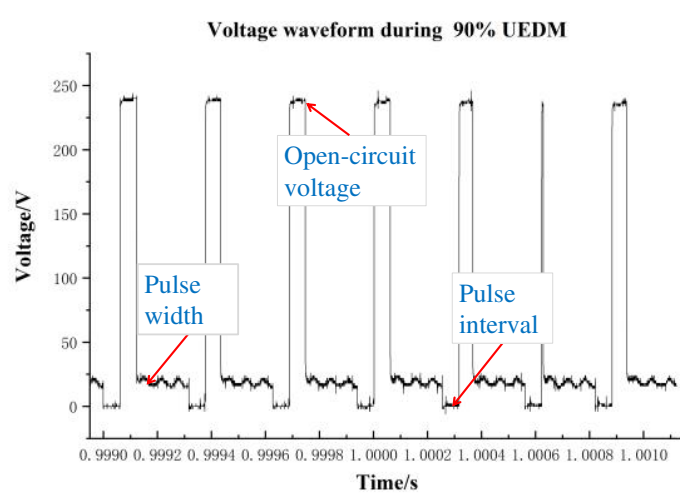

d) UEDM of $90 \%$ ultrasonic power specific voltage waveform

Fig.7 Different ultrasonic power than the voltage waveform

During the EDM processing, the electric split frequency is about $5 \mathrm{kHz}$. When the open circuit voltage breaks through the discharge medium, the electrodes start to discharge. The discharge voltage is relatively stable, as shown in Fig. 7a).

When the ultrasonic vibration with a frequency of $20 \mathrm{kHz}$ and a power ratio of $30 \%$ is applied, the regular sinusoidal variation of the voltage waveform on the basis of the EDM voltage waveform can be observed, as shown in Fig.7b). With the increase of ultrasonic power ratio, the inter-electrode voltage change increases, accompanied by unstable voltage mutation, as shown in Fig.7c). When the ultrasonic power ratio increases to $90 \%$, while the voltage amplitude increases, the unstable voltage also increases, and even a pull-off leads to re-breakdown discharge.

According to the analysis of the reason, in the process of ultrasonic shaking table vibration, the high-frequency ultrasonic vibration constantly changes the discharge gap, and the high-frequency change of the gap leads to the change of discharge voltage, which makes the discharge voltage change with the change of ultrasonic amplitude. The period of the change of discharge voltage is consistent with the frequency of ultrasonic vibration. In addition, in the process of increasing ultrasonic amplitude, the discharge gap will increase instantly, which will cause the discharge channel to be broken and then re-establish the discharge channel instantly. Therefore, it can be concluded that the lower amplitude ultrasonic vibration will cause the continuous change of voltage, and when the ultrasonic amplitude reaches a certain value, it will lead to the instantaneous collapse and reconstruction of the discharge channel.

\subsection{Analysis of Material Removal Surface Mechanism}

\subsubsection{Analysis of MRR}

In the actual processing process of UEDM, the material removal rate can not be measured accurately due to the small processing depth, which is transformed into the material removal time of removing the same depth pit, and the material removal rate is compared. The material removal rate results are shown in Fig.8. 


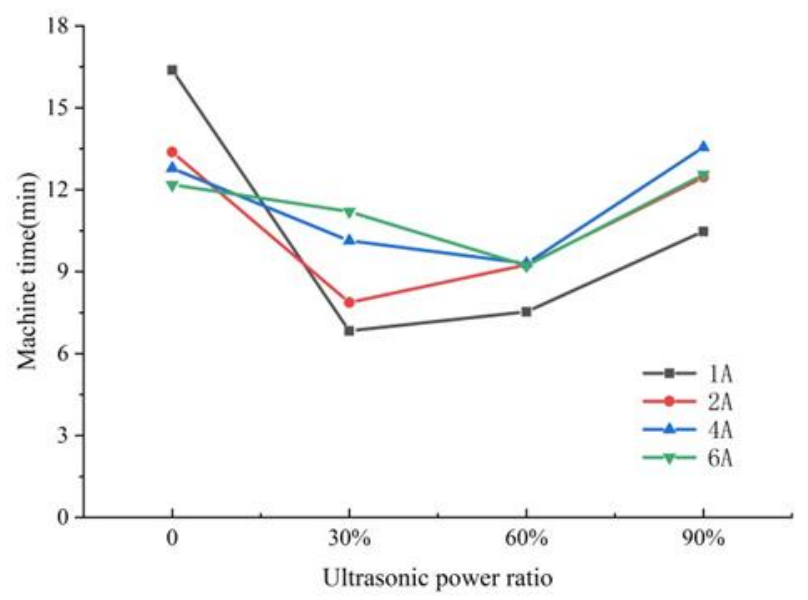

Fig.8 MRR under different ultrasonic power ratio parameters

As can be seen from Fig.8, the material removal rate of the workpiece increases at first and then decreases with the increase of ultrasonic power ratio, but in low current machining ( $\mathrm{I} \leq 2 \mathrm{~A}$ ), the material removal rate is the highest when the ultrasonic power ratio is $30 \%$. When the current is greater than $4 \mathrm{~A}$, when the ultrasonic power ratio is $60 \%$, the material removal rate of the workpiece is the highest. Compared with the material removal rate of ultrasonic vibration applied by different current, it can be concluded that the effect of ultrasonic vibration on EDM is more obvious when the current is small.

According to the analysis of the reason, in the actual machining, with the increase of the discharge current, the maximum discharge gap increases during the discharge period [11]. In small current machining, the discharge gap is small, and when ultrasonic vibration of $30 \%$ ultrasonic power ratio is applied, the discharge voltage vibrates in the maximum discharge gap, which changes the discharge voltage, and the discharge channel is not broken. However, when the ultrasonic power ratio increases to more than $60 \%$, the ultrasonic amplitude increases, which exceeds the maximum discharge gap of EDM, and the discharge channel is broken and re-established. The decrease of single discharge energy leads to the decrease of machining efficiency, so when ultrasonic vibration with $30 \%$ ultrasonic power ratio is applied, the material removal rate is the highest. When the current increases to more than $4 \mathrm{~A}$, the maximum discharge gap increases, and when $60 \%$ ultrasonic power ratio is applied, the discharge voltage still vibrates in the maximum discharge gap, so the corresponding material removal rate is the best.

\subsubsection{Surface Topography Analysis}

By observing the surface morphology of the workpiece treated by scanning electron microscope (SEM) under different ultrasonic power ratios, the micro-morphology of the workpiece surface is analyzed as shown in Fig.9. 


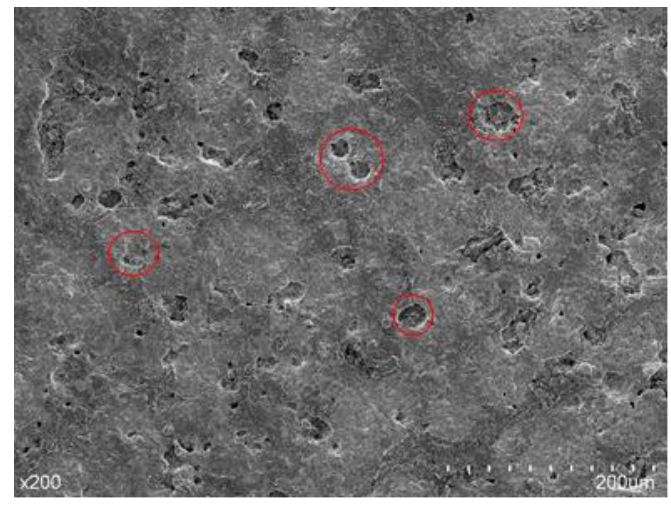

a) EDM surface

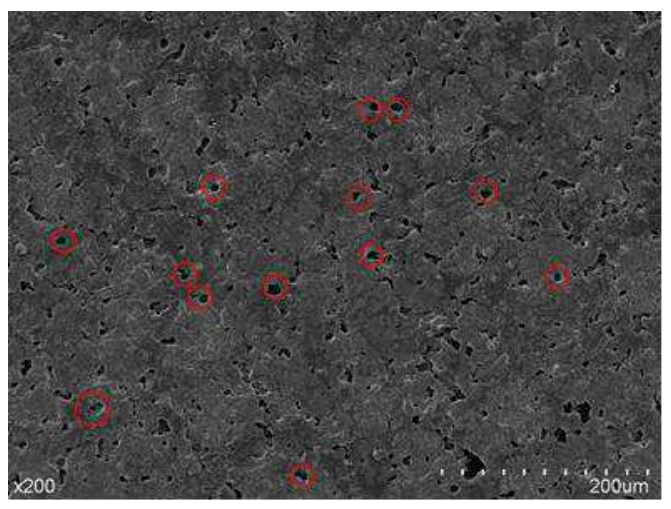

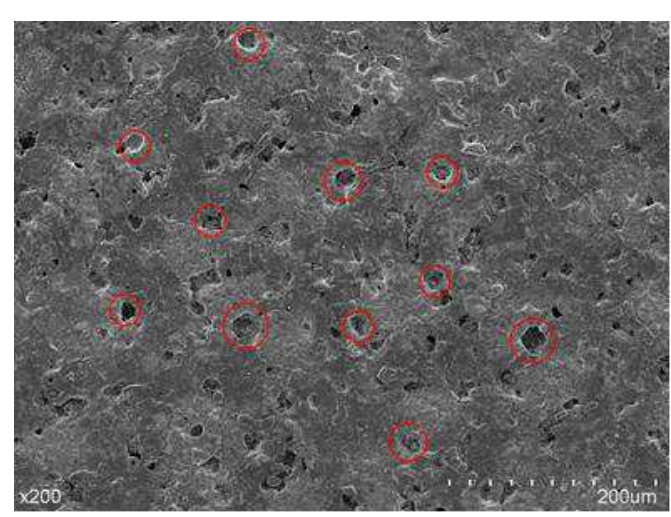

b) $30 \%$ ultrasonic power ratio of UEDM surface

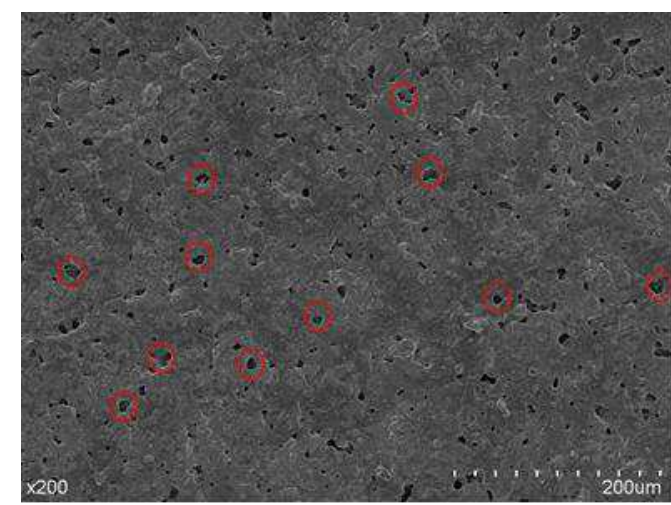

c) $60 \%$ ultrasonic Power ratio of UEDM surface d) $90 \%$ ultrasonic power ratio of UEDM surface

Fig.9 Surface topography under different ultrasonic power ratio parameters

The micro-morphology of the surface after EDM treatment in Fig.9a) shows that the pits obtained by EDM discharge are generally larger than 100um. After applying 30\% ultrasonic power ratio vibration, although the pits obtained by discharge are still generally larger than 100um, the number of discharge pits increases. When the ultrasonic power ratio increases by more than $60 \%$, it can be seen that most of the discharge pits are much less than 100um, and the number of tiny pits increases obviously, and the surface is gradually smooth with the increase of amplitude.

The results show that the introduction of ultrasonic vibration can improve the discharge probability, and under the influence of ultrasonic cavitation of low amplitude ultrasonic vibration. The recasting area is reduced and the machined surface is more smooth. However, with the increase of ultrasonic amplitude, the discharge channel is broken and re-established, which is equivalent to dividing the discharge pulse of a cycle into several pulses in an instant. In the study of Guo et al., the diameter and depth of the discharge pit decreased with the decrease of the pulse width [17]. Therefore, when the ultrasonic power ratio increased to more than $60 \%$, the discharge pit diameter suddenly changed. At the same time, the processing efficiency is relatively reduced.

\section{Conclusions}

In this paper, based on the theoretical analysis of voltage change in UEDM process, the energy model of UEDM discharge process is established, and the thermal process of material removal is described by heat conduction theory. Through experimental 
verification, the influence of ultrasonic vibration on EDM machined surface is obtained, and the following conclusions can be drawn:

1. Based on the change of gap voltage during UEDM, the mathematical model of UEDM discharge process is established, and the thermal process of material removal is described by heat conduction theory, and the removal process of single-pulse discharge material of EDM and UEDM is simulated. It is concluded that the center temperature of UEDM is higher than that of EDM, and increases with the increase of amplitude.

2. Through the experiments of EDM and UEDM, it can be found that the application of ultrasonic vibration can cause the change of voltage and then the change of material removal rate; in addition, the application of ultrasonic vibration can increase the discharge frequency of EDM, and with the increase of ultrasonic amplitude, it will cause the collapse and reconstruction of the discharge channel, reduce the single discharge energy, and produce more dense holes on the machined surface.

3. The effect of ultrasonic vibration on small and medium current machining of EDM is more obvious, under the condition of the same ultrasonic power ratio, the material removal rate of small current is higher; when machining with high current, in order to improve the machining efficiency, it is necessary to improve the ultrasonic vibration power ratio at the same time.

\section{Declaration}

\section{Funding}

This work supported by Natural Science Foundation of Tianjin City (18JCYBJC88900, 18JCQNJC05200, 18JCQNJC75300), National Natural Science Foundation of China (51505334).

\section{Competing Interest}

The authors declare that they have no conflicts of interest.

\section{Availability of data and materials}

All data generated or analyzed during this study are included in this published article.

\section{Authors Contributions}

All authors contributed to the study conception and design. Material preparation, data collection and analysis were performed by Yinghuai Dong, Jiajun Liu, Guangyan Li and Yan Wang. The first draft of the manuscript was written by Jiajun Liu and all authors commented on previous versions of the manuscript. All authors read and approved the final manuscript.

\section{Ethical Approval}

Not applicable

\section{Consent to Participate}

Not applicable

\section{Consent to Publish}

Not applicable

\section{References}

[1]. Wang, Y (2020) Analysis of material removal and surface generation mechanism of ultrasonic Vibration-assisted EDM. The International Journal of Advanced Manufacturing Technology 110(1-2): 177-189. 
[2] Yang XX (2015) Principle and process Application of EDM. National Defense Industry Press, Beijing

[3] Kremer D, Lebrun J L, Hosari B (1989) Effects of Ultrasonic Vibrations on the Performancesin EDM. Annals of the CIRP 38(1): 199-202.

[4] Kremer D, Lhiaubt C, Moisan A (1991) A Study of the Effect of Synchronizing Ultrasonic Vibrations with Pulses in EDM . Annals of the CIRP 40(1): 211-214.

[5] Lin, Y.C., Yan B.H., and Chang Y.S (2000) Machining characteristics of titanium alloy (Ti$6 \mathrm{Al}-4 \mathrm{~V}$ ) using a combination process of EDM with USM. Journal of materials processing technology 104(3): 171-177.

https://doi.org/10.1016/S0924-0136(00)00539-2

[6] Abdullah A, Shabgard M R (2008) Effect of ultrasonic vibration of tool on electrical discharge machining of cemented tungsten carbide (WC-Co). International Journal of Advanced Manufacturing Technology 38(11-12): 1137-1147.

https://doi.org/10.1007/s00170-007-1168-8

[7] Abdullah A, Shabgard M R, Ivanov A (2009) Effect of ultrasonic assisted EDM on the surface integrity of cemented tungsten carbide (WC-Co). International Journal of Advanced Manufacturing Technology 41(3-4):268-280.

https://doi.org/10.1007/s00170-008-1476-7

[8] Shabgard M R, Alenabi H (2015) Ultrasonic Assisted Electrical Discharge Machining of Ti-6Al-4V Alloy . Materials \& Manufacturing Processes 30 (8): 991-1000.

https://doi.org/10.1080/10426914.2015.1004686

[9] Liu, Y (2018) A Simulation Study of Debris Removal Process in Ultrasonic Vibration Assisted Electrical Discharge Machining (EDM) of Deep Holes. Micromachines 9(8): 378.

https://doi.org/10.3390/mi9080378

[10] Choubey, M., Maity K.P. and Sharma A (2020) Finite element modeling of material removal rate in micro-EDM process with and without ultrasonic vibration. Grey Systems: Theory and Application 10(3): 311-319.

https://doi.org/10.1108/GS-11-2019-0047

[11] Li, Z. and Bai J (2017) Impulse discharge method to investigate the influence of gap width on discharge characteristics in micro-EDM. The International Journal of Advanced Manufacturing Technology 90(5-8): 1769-1777.

https://oi.org/10.1007/s00170-016-9508-1

[12] Giridharan A, Samuel GL (2015) Modeling and analysis of crater formation during wire electrical discharge turning (WEDT) process. Int J Adv Manuf Technol 77(5-8):1229-1247.

https://doi.org/10.1007/s00170-014-6540-x

[13]Dibitoto, D.D., Eubank, P.T., Patel, M.R. and Barrufet, M.A (1989) Theoretical models of the electrical discharge machining process. I. A simple cathode erosion model. J. Appl. Phys., Vol. 66, Num. 9, pp 4095 - 4103.

[14] Shabgard, M (2013) Mathematical and numerical modeling of the effect of input-parameters on the flushing efficiency of plasma channel in EDM process. International Journal of Machine Tools and Manufacture 65: 79-87.

https://doi.org/10.1016/j.ijmachtools.2012.10.004

[15] Ikai T, Fujita I, Hashiguchi K (1995) Heat input for crater formationin EDM. Proceedings of International Symposium for Electro Machining-ISEMXI. EPFL, Lausanne, Switzerland, pp163- 
170.

[16] Joshi, S.N. and Pande S.S (2009) Development of an intelligent process model for EDM. The International Journal of Advanced Manufacturing Technology 45(3-4): 300-317.

https://doi.org/10.1007/s00170-009-1972-4

[17] Guo CH (2016) Research on establishment and simulation of temperature field model based on single pulse dry EDM. Dissertation, Harbin Institute of Technology 
Figures:
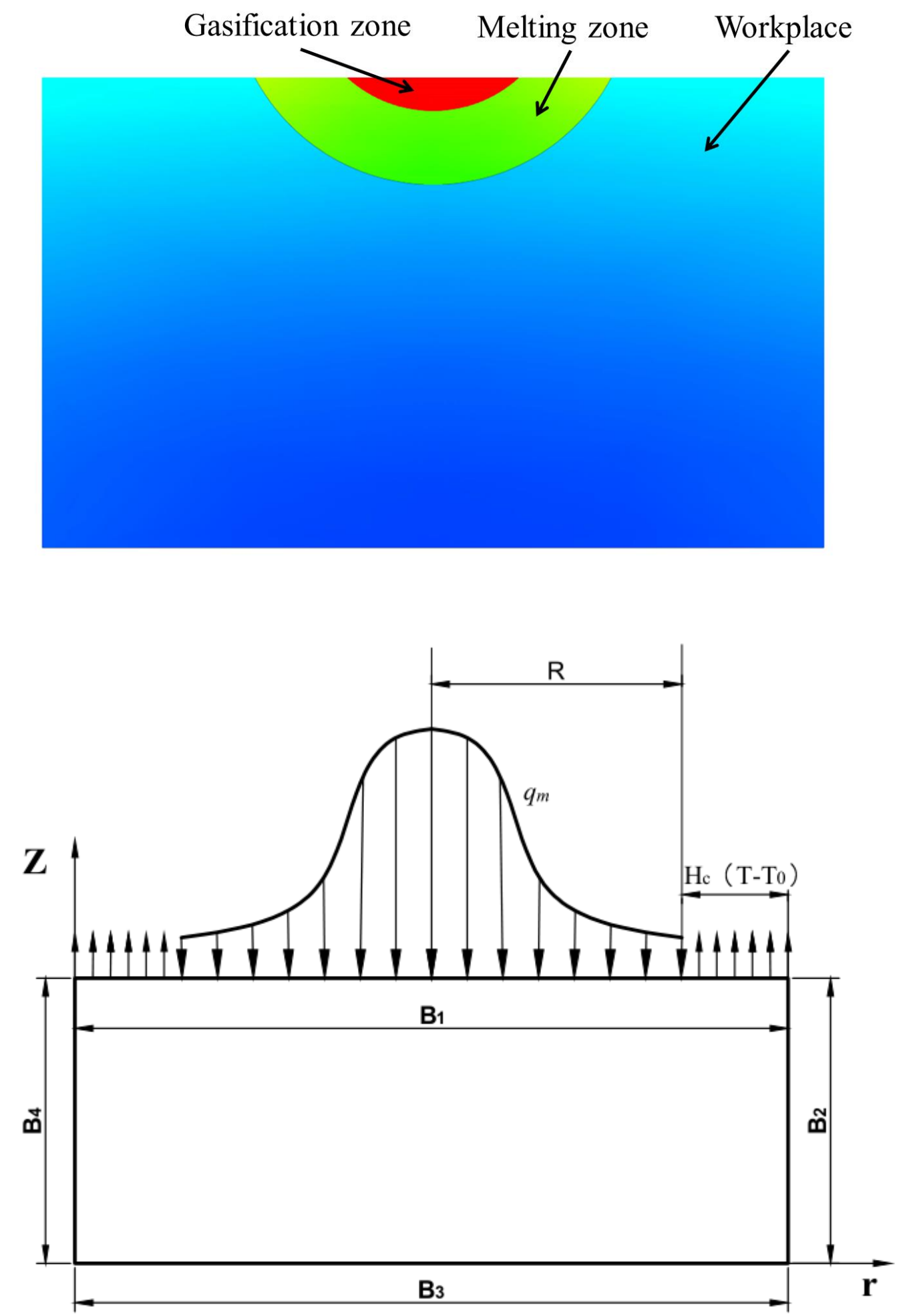


\section{B: EDM}

Temperature

Type: Temperature Unit: ${ }^{\circ} \mathrm{C}$

Time: 5.e-005

Custom

Max: 5773.3

Min: 25

5773.3

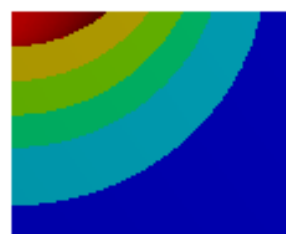

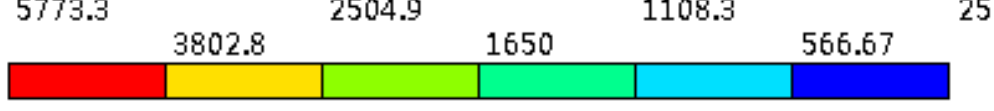

\section{B: EDM}

Temperature

Type: Temperature

Unit: ${ }^{\circ} \mathrm{C}$

Time: 5.e-005

Custom

Max: 5773.3

Min: 25

$2021 / 1 / 27$ 星期三 21:45

5773.3

2504.9

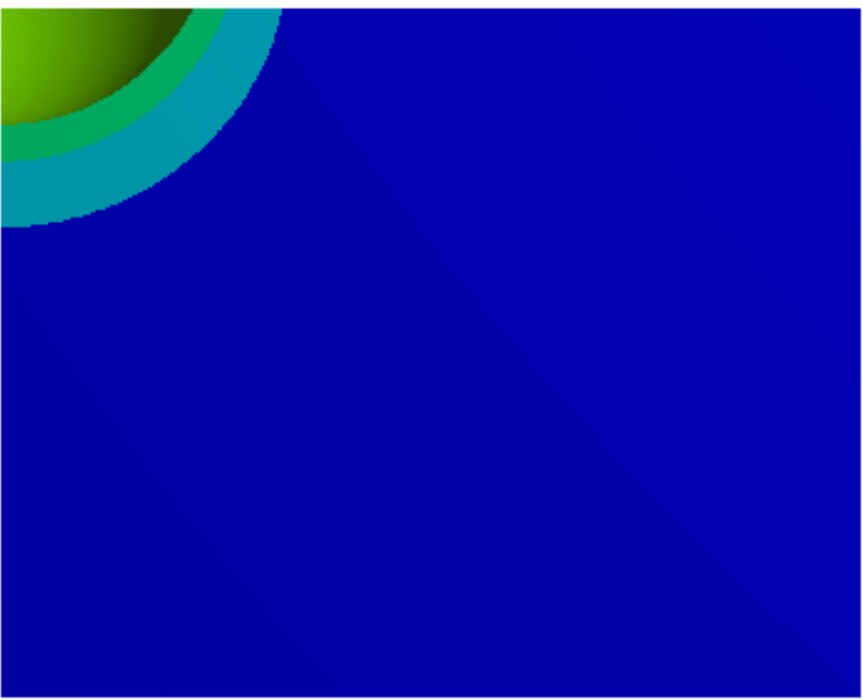

3802.8

$\begin{array}{lr}1650 & 566.7\end{array}$




\section{C: UEDM-1}

Temperature

Type: Temperature

Unit: ${ }^{\circ} \mathrm{C}$

Time: 5.e-005

Custom

Max: 7399.6

Min: 25

7399.6

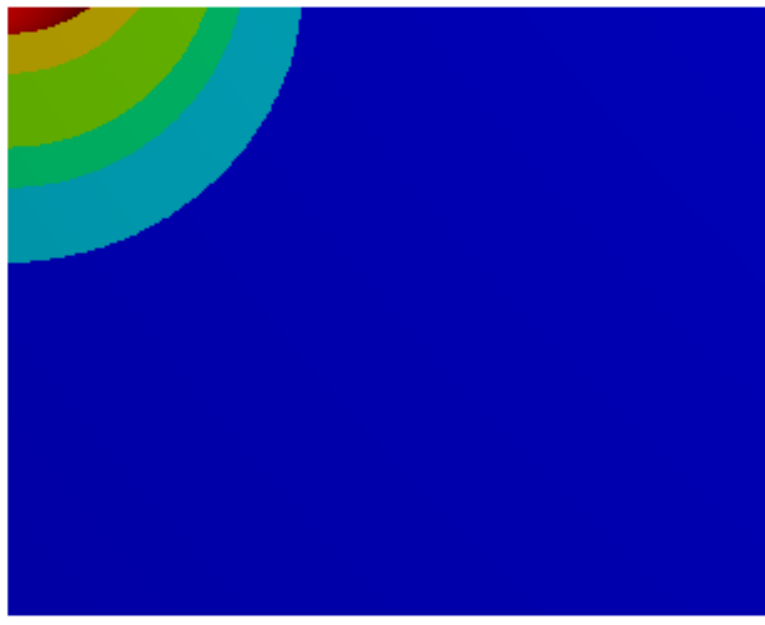

1108.3

566.67

25

\begin{tabular}{|l|l|l|l|l|}
\multicolumn{2}{|c}{5483.1} & \multicolumn{4}{c}{1650} & 566.67 \\
\hline & & & & \\
\end{tabular}

C: UEDM-1

Temperature

Type: Temperature

Unit: ${ }^{\circ} \mathrm{C}$

Time: 5.e-005

Max: 7399.6

Min: 25

2021/1/27 星期三 21:46

$7399.6 \quad 3566.5$

5483.1
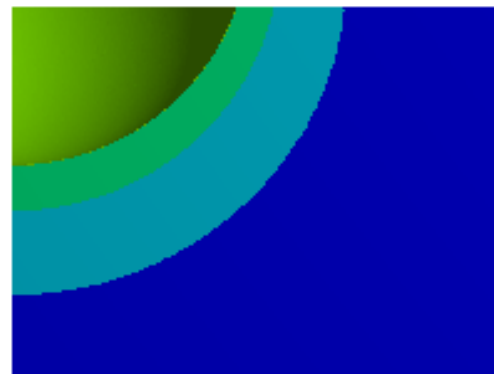


\section{D: UEDM-2}

Temperature

Type: Temperature

Unit: ${ }^{\circ} \mathrm{C}$

Time: 5.e-005

Custom

Max: 8268.3

Min: 25

8268.3

3856.1

1108.3

566.67

\section{D: UEDM-2}

Temperature

Type: Temperature

Unit: ${ }^{\circ} \mathrm{C}$

Time: 5.e-005

Custom

Max: 8268.3

Min: 25

8268.3

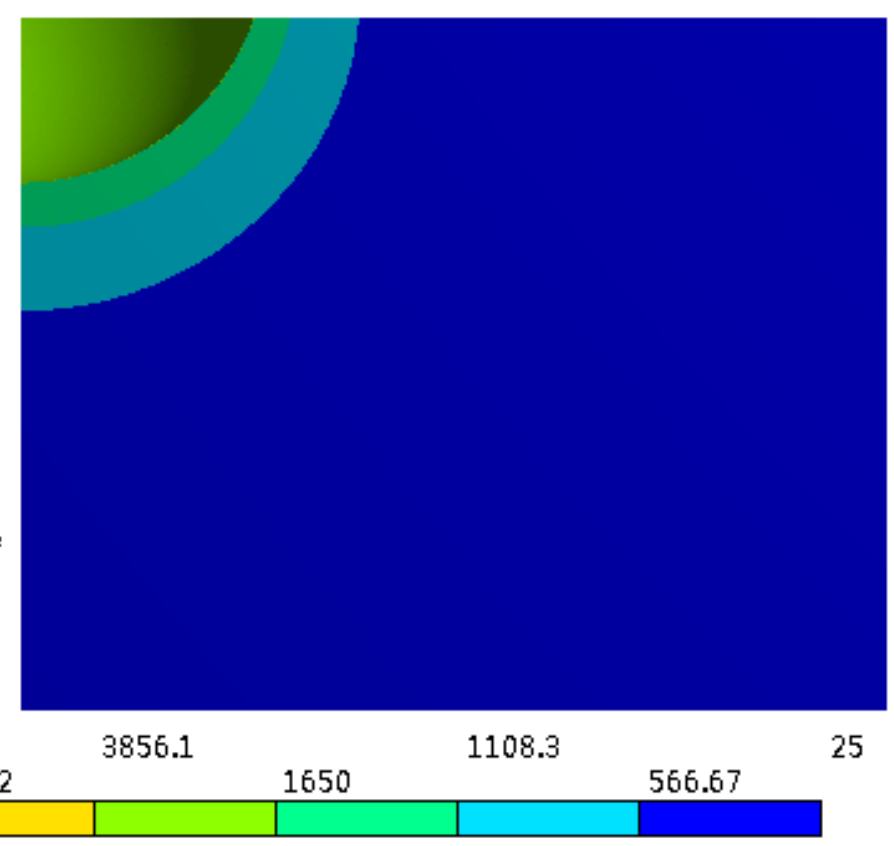



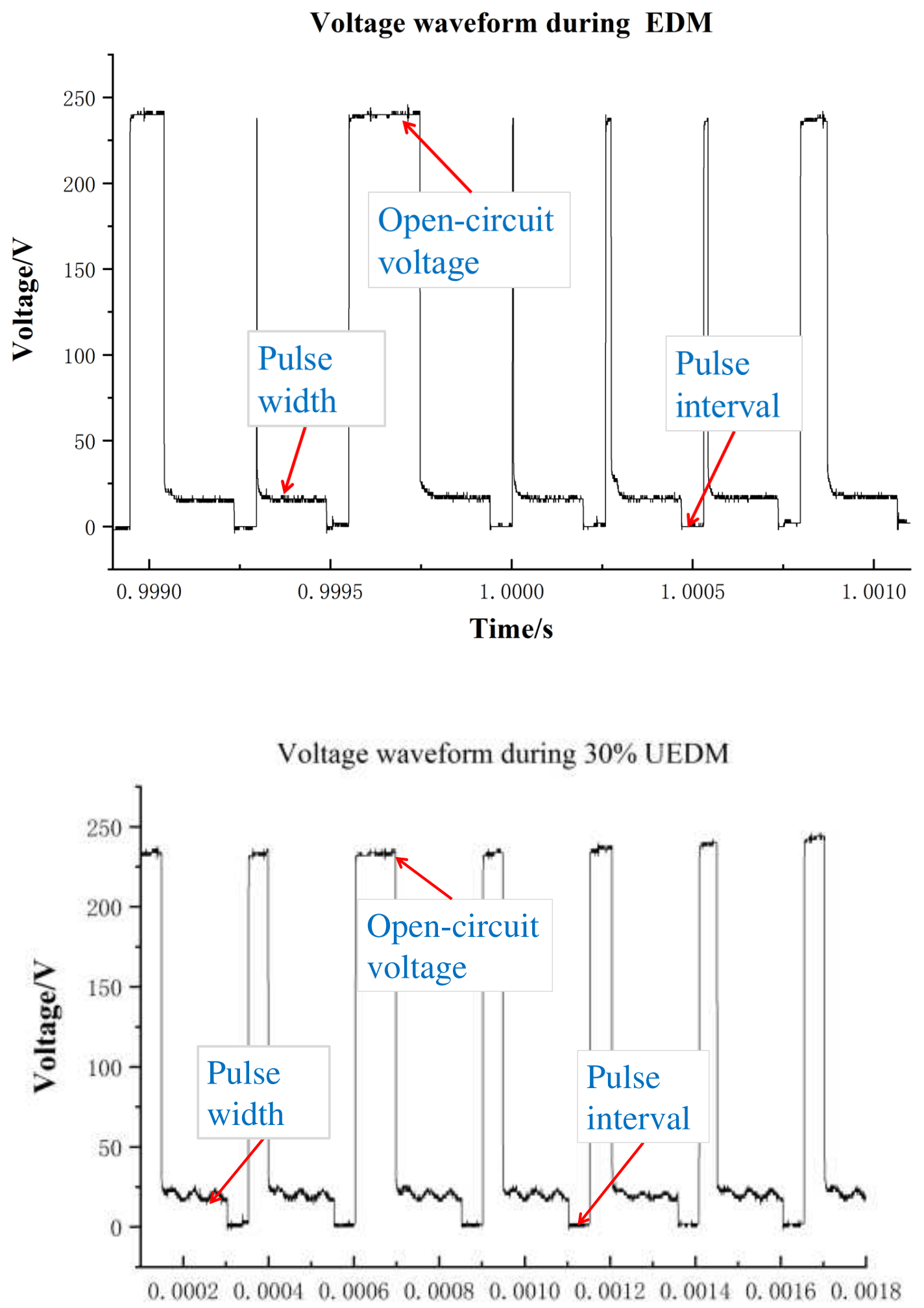

Time/s 

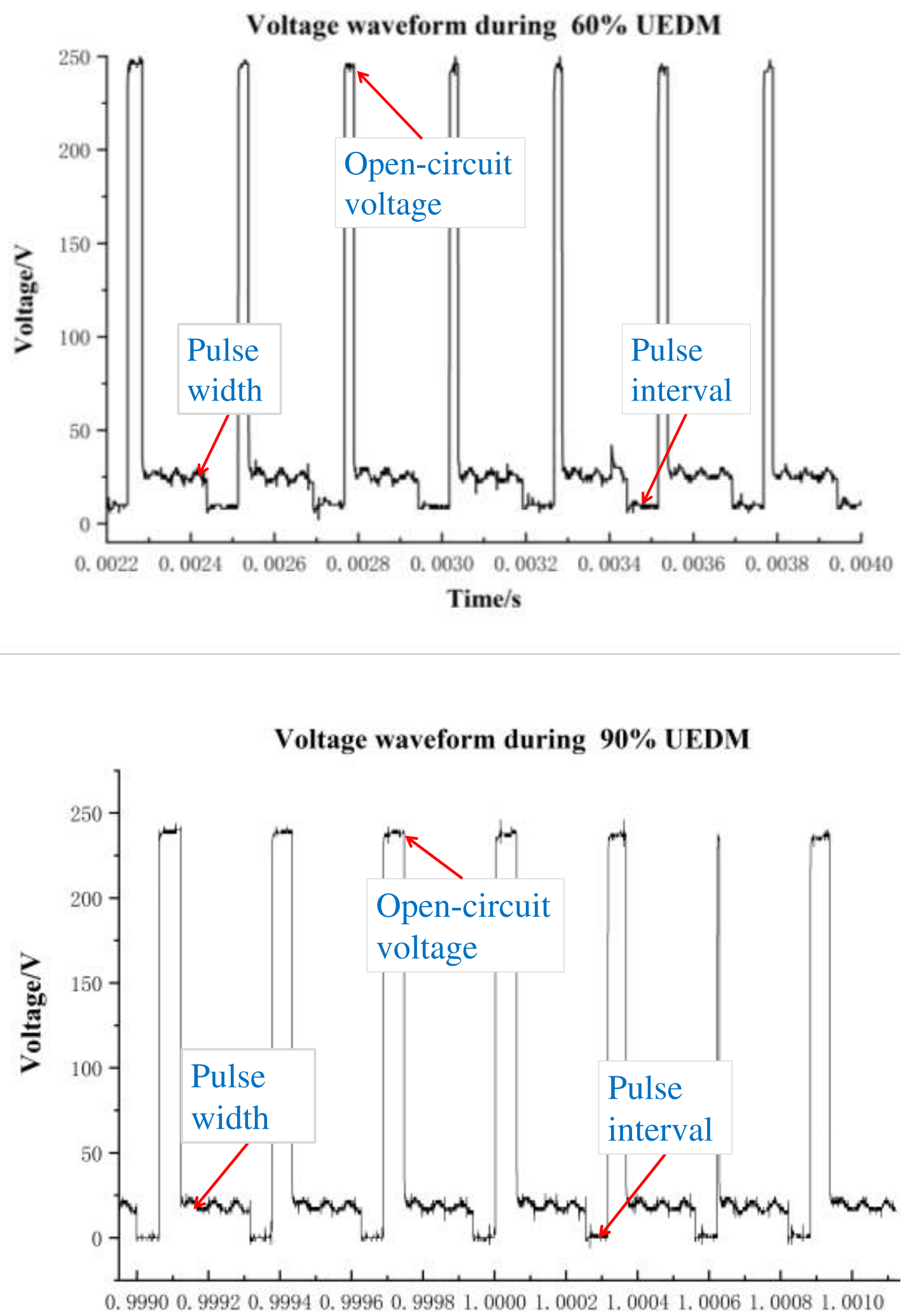

Time/s 

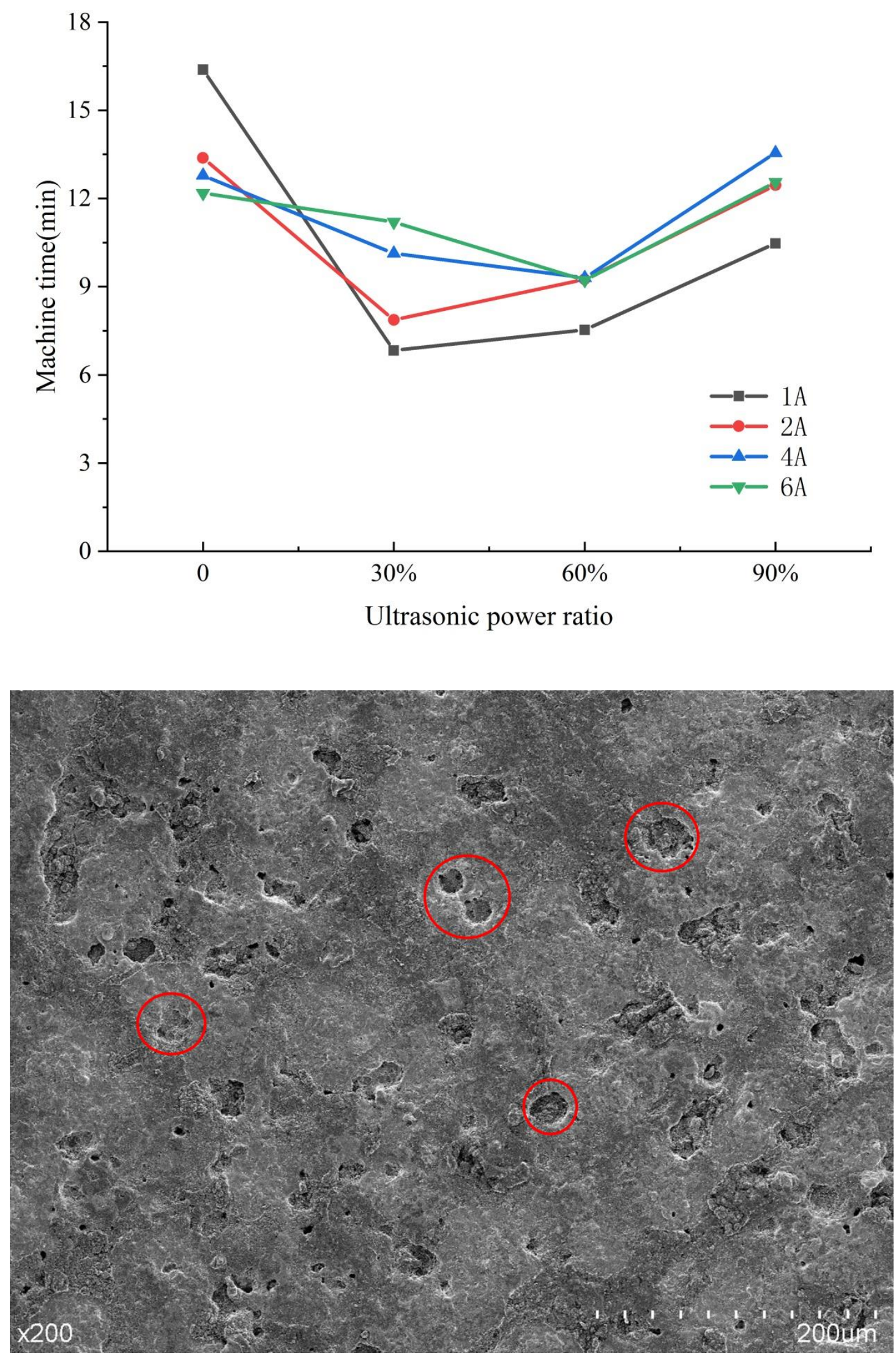

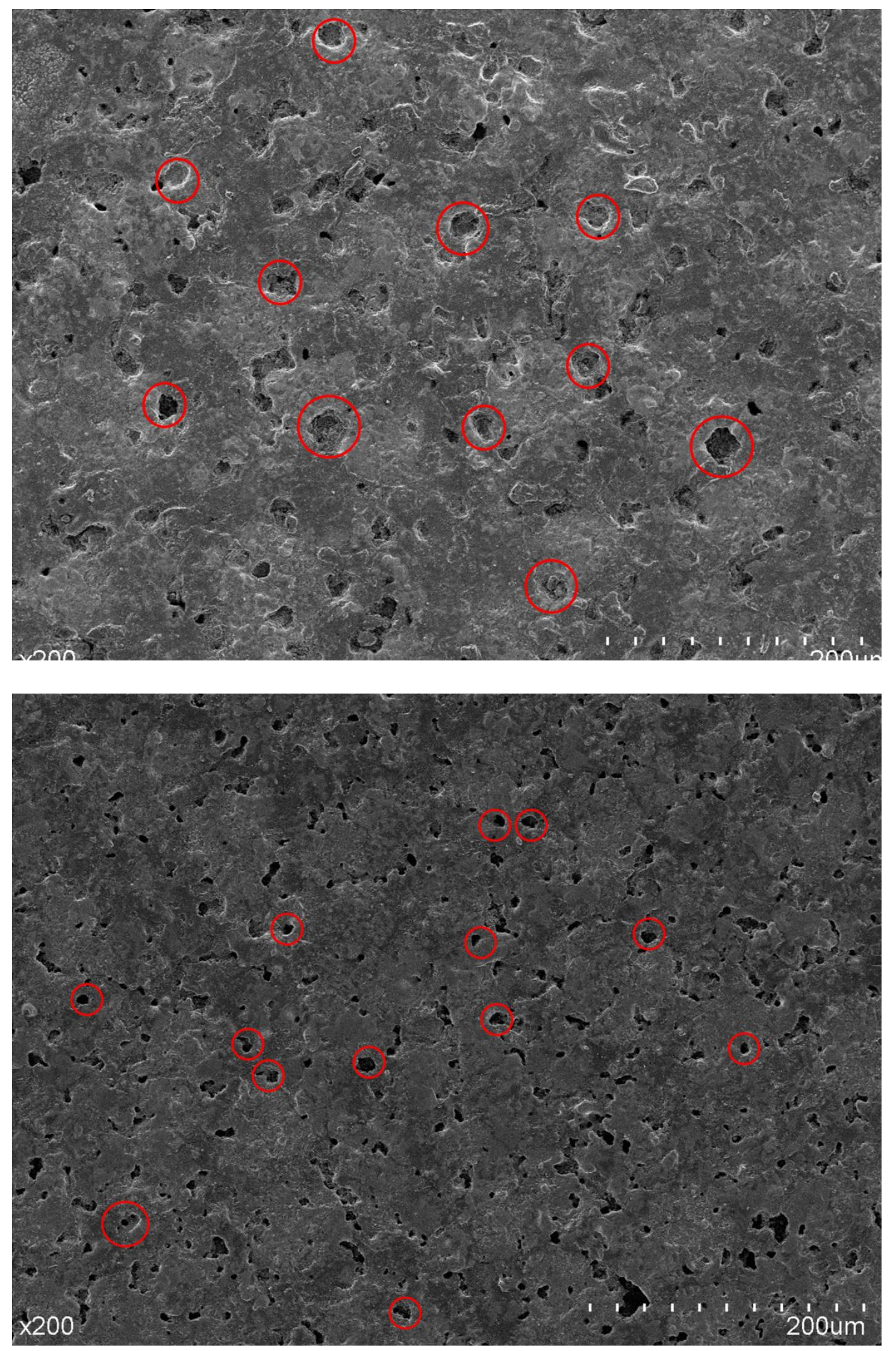


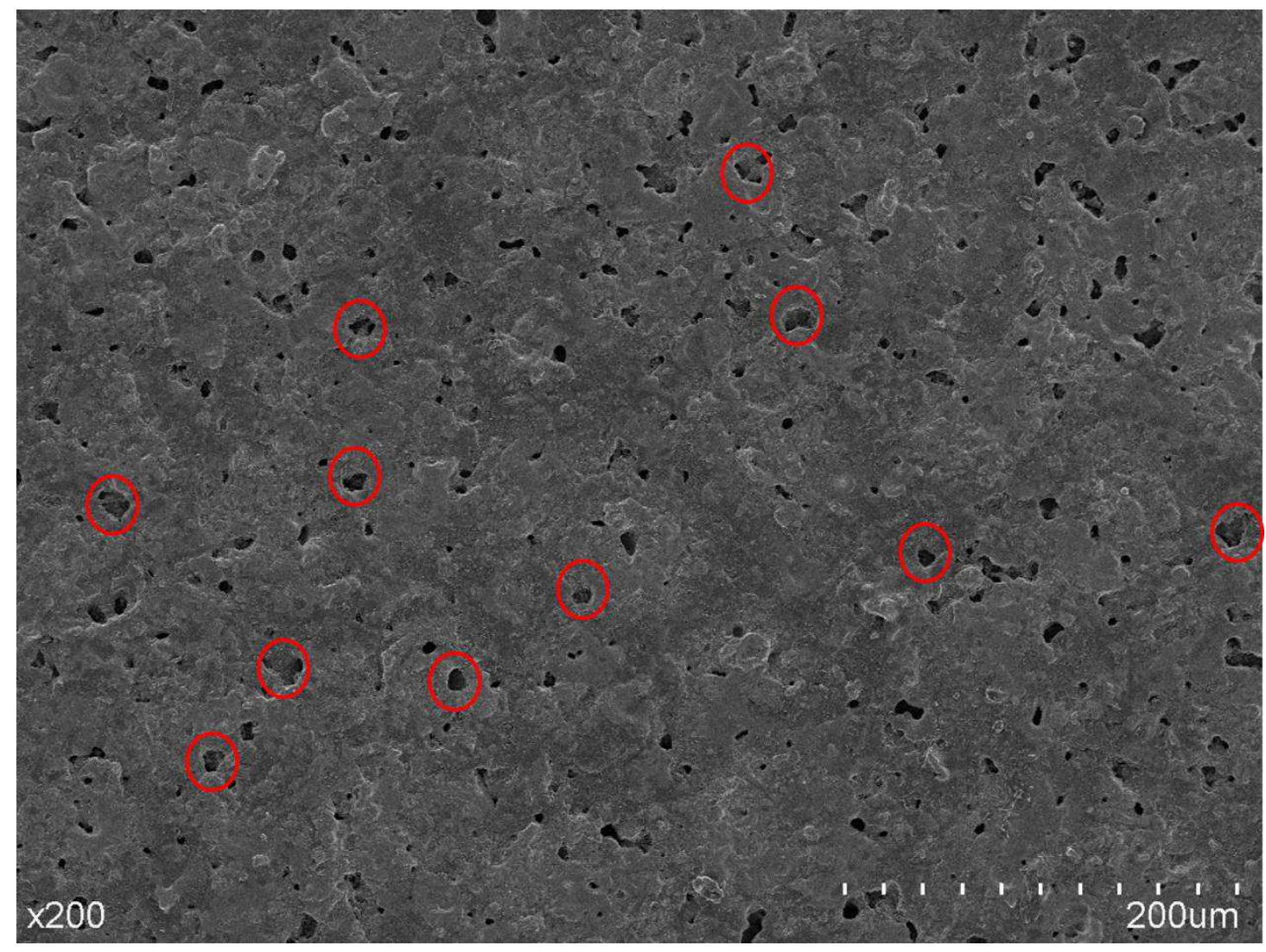

